\title{
Eight novel variants in the SLC34A2 gene in pulmonary alveolar microlithiasis
}

\author{
Åsa Lina M. Jönsson ${ }^{1}$, Elisabeth Bendstrup², Susie Mogensen', \\ Elizabeth J. Kopras ${ }^{3}$, Francis X. McCormack $\mathbb{1}^{3}$, Ilaria Campo $\mathbb{1}^{4}$ \\ Francesca Mariani ${ }^{4}$, Amparo Escribano-Montaner ${ }^{5}$, Are M. Holm ${ }^{6}$, \\ Maria del Mar Martinez-Colls ${ }^{7}$, Guillem Pintos-Morell ${ }^{7,8,9}$, Camille Taillé ${ }^{10}$, \\ Bruno Crestani ${ }^{10}$, Ole Hilberg ${ }^{2,11}$, Jane Hvarregaard Christensen ${ }^{1,12}$ \\ and Ulf Simonsen ${ }^{1,12}$
}

\begin{abstract}
Affiliations: ${ }^{1}$ Dept of Biomedicine, Aarhus University, Aarhus, Denmark. ${ }^{2}$ Dept of Respiratory Diseases and Allergy, Aarhus University Hospital, Aarhus, Denmark. ${ }^{3}$ Division of Pulmonary, Critical Care and Sleep Medicine, University of Cincinnati, Cincinnati, OH, USA. "Pneumology unit, IRCCS San Matteo Hospital Foundation, Pavia, Italy. ${ }^{5}$ Pediatric Pneumology Unit, Clinic University Hospital, Dept of Pediatrics, Obstetrics and Gynecology, University of Valencia, Valencia, Spain. ${ }^{6}$ Dept of Respiratory Medicine, Oslo University Hospital, Oslo, Norway. ${ }^{7}$ Dept of Pediatrics, University Hospital Germans Trias i Pujol, Badalona, Spain. ${ }^{8}$ Centre for Rare Diseases, University Hospital Vall d'Hebron, Barcelona, Spain. ${ }^{9}$ Research Institute IGTP, CIBERER-GCV08, Universitat Autònoma de Barcelona, Barcelona, Spain. ${ }^{10}$ APHP, Hopital Bichat, DHU FIRE, Pulmonology Dept, Reference Center for Rare Pulmonary Diseases, Unité Inserm 1152, Université Paris Diderot, Paris, France. ${ }^{11}$ Medical Dept, Vejle Hospital, Vejle, Denmark. ${ }^{12}$ Shared senior authorship.
\end{abstract}

Correspondence: Åsa Lina M. Jönsson, Dept of Biomedicine, Aarhus University, Bartholins Allé 6, Building 1242, 3rd Floor, 8000 Aarhus C, Denmark. E-mail: aasajoendarm.dk

@ERSpublications

Eight novel variants in the SLC34A2 gene have been identified in 14 patients with pulmonary alveolar microlithiasis (PAM), which emphasises the importance of the gene in the disease. Furthermore, a genotype-phenotype correlation in PAM may exist. http://bit.ly/3307M1p

Cite this article as: Jönsson ÅLM, Bendstrup E, Mogensen S, et al. Eight novel variants in the SLC34A2 gene in pulmonary alveolar microlithiasis. Eur Respir J 2020; 55: 1900806 [https://doi.org/10.1183/ 13993003.00806-2019].

\section{ABSTRACT}

Background: Pulmonary alveolar microlithiasis (PAM) is caused by genetic variants in the SLC34A2 gene, which encodes the sodium-dependent phosphate transport protein $2 \mathrm{~B}(\mathrm{NaPi}-2 \mathrm{~b})$. PAM is characterised by deposition of calcium phosphate concretions (microliths) in the alveoli leading to pulmonary dysfunction. The variant spectrum of SLC34A2 has not been well investigated and it is not yet known whether a genotype-phenotype correlation exists.

Methods: We collected DNA from 14 patients with PAM and four relatives, and analysed the coding regions of SLC34A2 by direct DNA sequencing. To determine the phenotype characteristics, clinical data were collected and a severity score was created for each variant, based on type and localisation within the protein. Results: We identified eight novel allelic variants of SLC34A2 in 14 patients with PAM. Four of these were nonsense variants, three were missense and one was a splice site variant. One patient was heterozygous for two different variants and all other patients were homozygous. Four patients were asymptomatic and 10 patients were symptomatic. The severity of the disease was associated with the variant severity.

Conclusions: Our findings support a significant role for SLC34A2 in PAM and expand the variant spectrum of the disease. Thus, SLC34A2 variants were detected in all patients and eight novel allelic variants were discovered. An association between disease severity and the severity of the variants was found; however, this needs to be investigated in larger patient populations.

This article has supplementary material available from erj.ersjournals.com

Received: 22 April 2019 | Accepted after revision: 15 Nov 2019

Copyright OERS 2020 


\section{Introduction}

Pulmonary alveolar microlithiasis (PAM) is a rare inherited lung disease with less than 1100 patients reported worldwide since 1933. The disease is characterised by deposition of calcium phosphate concretions (microliths) in the alveoli of the lungs [1]. Symptoms are dyspnoea, nonproductive cough, chest pain and fatigue [2]. The clinical course is variable; in some patients, the disease remains relatively quiescent while in others it progresses to respiratory insufficiency and early death [1]. Lung transplantation is currently the only existing effective treatment [1].

PAM is considered to be an autosomal recessive disorder with high penetrance (OMIM \#265 100). The only known causative gene is solute carrier family 34 member 2 (SLC34A2) (Entrez Gene ID 10568) [1, 3-5]. It is located on the short arm of chromosome four (4p15.2) and it has 13 exons, of which the first is noncoding. SLC34A2 encodes a 690 amino acid protein, the sodium-dependent phosphate transport protein $2 \mathrm{~B}(\mathrm{NaPi}-2 \mathrm{~b})$, which plays a role in inorganic phosphate homeostasis [5-7]. SLC34A2 is expressed in type II alveolar cells [4, 8], which play an important role in surfactant recycling and catabolism [9], and possibly also in the export from the alveolar space of phosphate liberated from degraded phospholipids. In PAM, dysfunction of NaPi-2b due to genetic variants in SLC34A2 might therefore cause a defect in cell uptake of phosphate, leading to elevation of phosphate levels in the alveolar lining fluid and deposition of calcium phosphate concretions in the alveoli $[4,10]$.

Since the identification of the first genetic variants in PAM patients in 2006, a total of 22 different allelic variants have been reported in less than 50 patients (figure 1). The most common DNA alterations in SLC34A2 involve changes to single or a few nucleotides, but more complex variants have been described including combinations of deletion-insertions and larger deletions $[4,17,18]$. The variants are proposed to lead to protein truncation with a possible decreased protein activity or to gene expression silencing $[1,3,4,19,20]$. Furthermore, elimination of the mRNA due to nonsense-mediated decay is another possible outcome [16]. However, no detailed review of clinical data in PAM patients with detected genetic variants has so far been reported. Therefore, no assessments of association between the genotype and the severity of the pulmonary and extrapulmonary symptoms have been made.

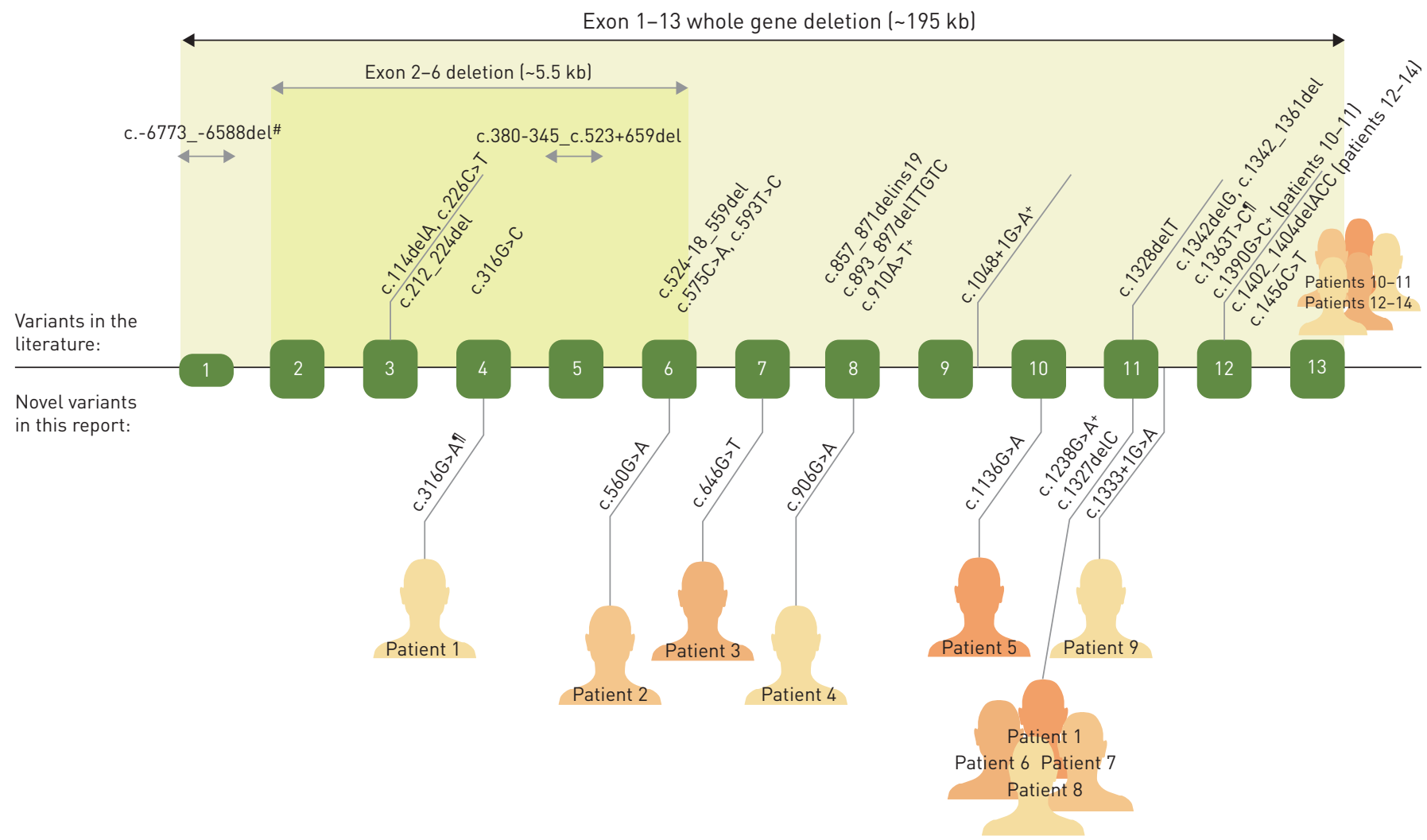

FIGURE 1 Allelic variants in SLC34A2 in patients with pulmonary alveolar microlithiasis (PAM) in the literature [2-4, 11-23] and novel variants presented in this study. Patients 13 and 14 are previously described with the variant c.1402_1404delACC [2]. Variants are present in homozygous form unless otherwise stated. Narrow boxes are for noncoding exons and wider boxes are for coding exons. Exons, introns and deletions are not drawn to scale. " : involving the SLC34A2 promoter region; " ${ }^{\text {: }}$ compound heterozygous; ${ }^{+}$: homozygous and compound heterozygous. 
The specific objectives of our study were to expand the allelic spectrum of SCL34A2 in patients with PAM and to evaluate a possible genotype-phenotype correlation. We present the genotype and phenotype characteristics in a series of PAM patients and contribute important new knowledge of the variant spectrum of SLC34A2. In addition, we investigate the correlation between the clinical phenotype and the localisation of the genetic variant and variant type.

\section{Patients and methods}

Patients

Fourteen patients with PAM and four relatives were included in this case series, from seven medical centres between 2014 and 2017. The clinical diagnosis of PAM was based on either radiographic appearance, a typical histological picture in lung biopsy, or the presence of microliths in bronchoalveolar lavage fluid (BALF). The project protocol was approved by the Central Denmark Region Committees on Biomedical Research Ethics (1-10-72-10-14). Sampling was performed according to clinical practice and the regulations in each country, complying with the principles of the Declaration of Helsinki. Written informed consent was obtained from the subjects.

\section{Sampling and data analyses}

Total DNA was extracted from venous blood (5-10 mL in EDTA) or saliva using standard protocols. Genetic analysis included direct DNA sequencing of PCR-amplified coding regions and flanking splice sites of the SLC34A2 gene (see supplementary material and supplementary table E1). Sequencing data was analysed using Mutation Surveyor Loc48 version 3.20 (SoftGenetics LLC, State College, PA, USA) with the SLC34A2 DNA sequence as a reference (Ensembl Transcript ID ENST00000382051.7 (GRCh38.p12 assembly)). We screened for the prior inclusion of all variants in public databases of known genetic variation, including the Genome Aggregation Database (GnomAD) [24], the Exome Variant Server, the NHLBI GO Exome Sequencing Project (ESP) [25], the 1000 Genomes Project [26] and the Database of Single Nucleotide Polymorphisms (dbSNP) [27], as well as through searches of the scientific literature [28] and the Human Gene Mutation Database (HGMD Professional 2019.1) [29]. Variants with a frequency in the general population above $0.1 \%$ were considered to be common and accordingly were not considered causative for PAM.

The severity of the disease was stated as a "clinical disease severity score" based on a composite measure of nine clinical parameters. Each parameter was scored between zero and one points (pulmonary hypertension $(\mathrm{PH})$, chest pain, fatigue, clinical progression, limitations of daily activities and incapability to work) or between zero and two points (forced vital capacity (FVC), diffusing capacity of the lung for carbon monoxide $\left(D_{\mathrm{LCO}}\right)$ and dyspnoea) (see supplementary table E2). The maximum obtainable score was 12 and it is presented as a ratio with a maximum average score of 1.33 . The severity of the disease was graded into three groups: mild (0-0.44), moderate (0.45-0.88) and severe (0.89-1.33).

The variants were interpreted in silico with MutationTaster2 (www.mutationtaster.org) [30], PANTHER version 13.1 (http://pantherdb.org) [31], Polyphen-2 version 2.2.2r398 (http://genetics.bwh.harvard.edu/ pph2/) [32], PROVEAN version 1.1.3. (http://provean.jcvi.org) [33] and Human Splicing Finder version 3.1 (www.umd.be/HSF3/) [34]. The variants were classified according to American College of Medical Genetics and Genomics (ACMG) and Association for Molecular Pathology (AMP) criteria [35]. In addition, the variants were classified into three severity groups: mild ( 0 points), moderate ( 1 point) and severe ( 2 points), on the basis of their predicted effect on $\mathrm{NaPi}-2 \mathrm{~b}$ function based on the variant type and localisation within the protein. Additional details regarding this classification are provided in the supplementary material.

\section{Statistical analysis}

The calculations were performed in Stata 11.2 (StataCorp 2009, College Station, TX, USA). Normality was inspected by QQ-plots of data. Pairwise correlation was tested with Spearman's rank correlation. A p-value of less than 0.05 was considered statistically significant.

\section{Results}

\section{Clinical findings}

Demographics and clinical characteristics

Table 1 and supplementary table E3 summarise the clinical characteristics and demographics of the patients. The mean \pm SEM age was $39.6 \pm 5.9$ years and age at diagnosis varied from 0 to 66 years. The median follow-up time was 12 years (range: 4-39 years). Patients 4, 8 and 10 were diagnosed with PAM in the setting of familial testing. In six patients, PAM was an incidental diagnosis based on chest radiographs taken for another purpose. The patients were from Denmark $(n=2)$, France $(n=1)$, Italy $(n=2)$, Norway $(n=2)$, Spain $(n=3)$ and the USA $(n=4)$. Patients were Caucasian $(n=13)$ or Arabian $(n=1)$. Seven patients had at least one known relative with PAM including two pairs of siblings (patients 7 and 8 as well as patients 10 and 11). Consanguinity was reported in three patients. 
TABLE 1 Clinical characteristics of the patients

\begin{tabular}{|c|c|c|c|c|c|c|c|c|c|c|c|}
\hline \multirow{2}{*}{$\begin{array}{l}\text { Patient } \\
\text { ID }\end{array}$} & \multirow[t]{2}{*}{ Sex } & \multicolumn{2}{|c|}{ Age years } & \multirow[t]{2}{*}{ Symptoms } & \multirow{2}{*}{$\begin{array}{c}\text { CT } \\
\text { involvement }\end{array}$} & \multirow{2}{*}{$\begin{array}{c}\text { FVC } \% \\
\text { predicted }\end{array}$} & \multirow{2}{*}{$\begin{array}{c}D_{\mathrm{Lco}} \% \\
\text { predicted }\end{array}$} & \multicolumn{2}{|c|}{ Microliths } & \multirow{2}{*}{$\begin{array}{l}\text { Clinical } \\
\text { course }^{\text {I }}\end{array}$} & \multirow[t]{2}{*}{ PAM specific therapy } \\
\hline & & Current & At diagnosis & & & & & In biopsy & In BALF & & \\
\hline 1 & $\mathrm{~F}$ & 39 & 22 & Dyspnoea, chest pain & No data & 93 & 63 & No data & Not performed & Stable & None \\
\hline 2 & $\mathrm{~F}$ & 9 & 5 & None & $>50$ & 89 & 63 & Not performed & Not performed & Stable & None \\
\hline 3 & $\mathrm{~F}$ & $-^{+}$ & 66 & $\begin{array}{l}\text { Dyspnoea, cough, } \\
\text { asthenia }\end{array}$ & No data ${ }^{\S}$ & 57 & 23 & No data & No data & Progress & $\begin{array}{c}\left.\text { ALD (70 mg } \cdot \text { week }^{-1}\right), \text { PSE } \\
\left(10 \mathrm{mg} \cdot \text { day }^{-1}\right)\end{array}$ \\
\hline 4 & $\mathrm{~F}$ & 40 & 34 & Dyspnoea & $>50^{f}$ & 80 & 51 & Yes & Yes & Progress & $\begin{array}{c}\text { Unknown effect } \\
\text { STS (12.5 g.month }{ }^{-1} ; 9 \text { months) } \\
\text { No effect }{ }^{\# \#}\end{array}$ \\
\hline 5 & M & 54 & 46 & None & $>50$ ๆศก & 92 & 60 & Yes & Yes & Progress & None \\
\hline 6 & $\mathrm{~F}$ & 37 & No data & Dyspnoea, cough & No data & No data & No data & No data & No data & No data & No data \\
\hline 7 & $\mathrm{~F}$ & $-^{++}$ & 20 & $\begin{array}{l}\text { Dyspnoea, chest pain, } \\
\text { asthenia }\end{array}$ & $>50$ & 52 & 38 & No data & Not performed & Progress & None \\
\hline 8 & $\mathrm{~F}$ & 52 & 23 & $\begin{array}{l}\text { Dyspnoea, chest pain, } \\
\text { asthenia, cough }\end{array}$ & $>50$ & 52 & 25 & No data & Not performed & Progress & None \\
\hline 9 & M & 58 & 19 & Dyspnoea, cough & $>50$ & No data & No data & Yes & Not performed & Progress & None \\
\hline 10 & $M$ & 9 & 9 months & None after age four $\S \S$ & $20-50$ & 102 & 87 & Not performed & Not performed & Stable & None \\
\hline 11 & $\mathrm{~F}$ & 14 & 5 & None after age four $\S \S$ & $>50$ & 94 & 74 & Yes & No data & Stable & None \\
\hline 12 & $\mathrm{~F}$ & 69 & $51^{f f}$ & $\begin{array}{c}\text { Dyspnoea, asthenia, } \\
\text { cough }\end{array}$ & No data & 45 & 29 & Not performed & Not performed & Progress & $\begin{array}{c}\text { LPD (2 years) } \\
\text { Serum phosphate decreased }\end{array}$ \\
\hline 13 & M & 32 & 16 & $\begin{array}{l}\text { Dyspnoea, chest pain, } \\
\text { asthenia, cough }\end{array}$ & $>50$ & No data & $\begin{array}{c}\text { No } \\
\text { data\#\#\# }\end{array}$ & Yes & No & Progress & $\begin{array}{c}\left.\text { EHDP (200 mg } \cdot \text { day }^{-1} ; 1.5 \text { years }\right) \\
\text { No effect }\end{array}$ \\
\hline 14 & M & 62 & 50 & Dyspnoea, asthenia & $>50$ & 87 & 33 & Yes & No & Progress & $\begin{array}{c}\text { PDN (2 months), EHDP } \\
\text { (200 mg.day }{ }^{-1} ; 6 \text { months) } \\
\text { No effect }\end{array}$ \\
\hline
\end{tabular}

CT: computed tomography; FVC: forced vital capacity; $D_{\mathrm{LCO}}$ : diffusing capacity of the lung for carbon monoxide; BALF: bronchoalveolar lavage fluid; PAM: pulmonary alveolar microlithiasis; ALD: alendronate; PSE: prednisone; STS: sodium thiosulfate; LPD: low phosphate diet; EHDP: disodium etidronate; PDN: prednisolone. " : CT involvement was based on a clinical estimation from each medical centre and was not standardised; ${ }^{\Uparrow}$ : clinical course was evaluated on symptoms, pulmonary function tests (PFTs) and radiographical appearance; ${ }^{+}$: patient was lost to follow-up at age 66; ${ }^{\S}$ : patient was known with parenchymal calcifications from age 10; $f_{\text {: }}$ extensive radiographic progression, slow clinical progression; $\#$ \# : treatment with STS in this patient was previously reported by TAlLLE et al. [36]; ${ }^{\text {กी }}$ : radiographic abnormalities detected at the age six; ${ }^{++}$: patient died at age 47 ; ${ }^{\S}$ : patient experienced respiratory symptoms including broncho-obstructive crises and recurrent pneumonias until the age of four, but has been asymptomatic since; ${ }^{f f}$ : patient was misclassified with

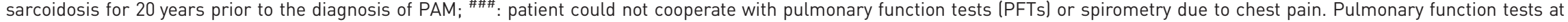
the time of diagnosis revealed signs of mild restrictive pattern and normal $D_{\text {LCo. }}$ 

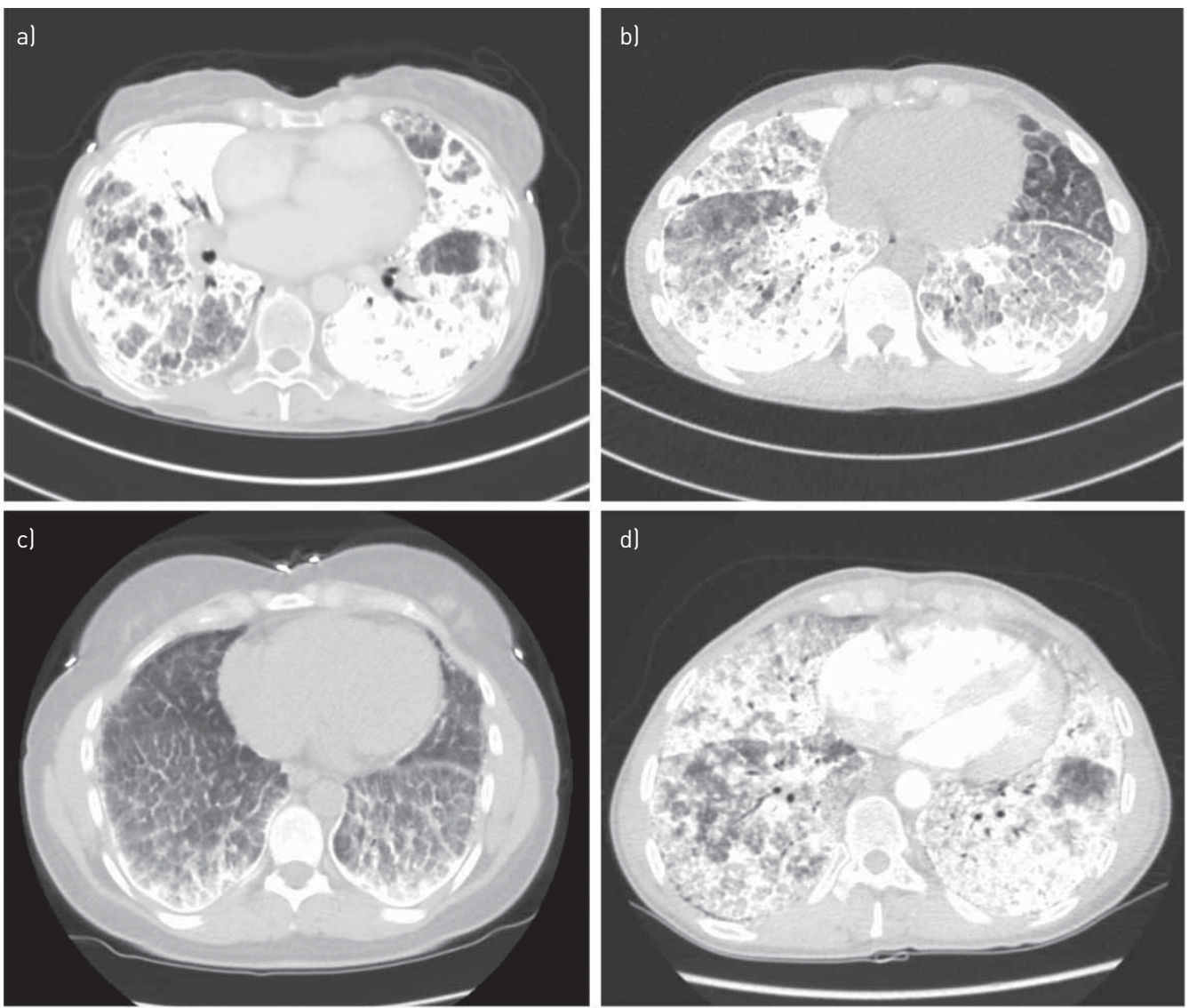

FIGURE 2 Computed tomography (CT) scans in pulmonary alveolar microlithiasis (PAM) patients showing different degrees of calcification with numerous sand-like calcifications, calcified interlobular septa and consolidations throughout the lungs with a subpleural localisation. Patient 12 (a) and patient 13 (b) are homozygous for the same genetic variant in SLC34A2 and present with similar degrees of calcification in the lungs. Different levels of severity of lung involvement are seen in patient 1 (c) (mild clinical disease) and patient 7 (d) (severe clinical disease).

The majority of patients reported symptoms typical for PAM, most commonly dyspnoea. The typical radiographic appearance of hyperdense infiltrates was seen in all patients on chest radiography, computed tomography (CT) scan and/or high-resolution computed tomography (HRCT) scan (figure 2). Pulmonary function tests (PFTs) revealed a restrictive pattern with impaired total lung capacity (TLC) for five out of 10 patients and decreased $D_{\mathrm{LCO}}$ for 10 out of 11 patients. The diagnosis was confirmed by histopathological examination of a lung biopsy (either a transbronchial biopsy or a surgical lung biopsy) in eight out of nine patients. In the majority of patients, blood levels of calcium and phosphate were within normal levels. Kidney and gallbladder stones were reported in three patients, one of which was additionally found to have calcifications in the gastric ventricular wall and was diagnosed in adolescence with a significant aortic valve stenosis (AS). Considerable comorbidity was reported in eight patients (see supplementary table E3).

Treatment and course of disease

In five patients, specific treatments for PAM had been attempted (table 1). Patient 12 was put on a low phosphate diet (LPD) for about 2 years and, after 4 months, their serum phosphate level had decreased from 1.26 to $1.00 \mathrm{mmol} \cdot \mathrm{L}^{-1}$; however, no clinical benefits were reported. PAM was progressive in nine patients and stable in four (with similar follow-up times in the two groups, $p=0.26$ ). One patient died shortly after inclusion in the study due to respiratory failure and two of the patients have previously been described in detail $[2,37]$. Both were reported symptomatic with decreased lung function and without treatment benefits. Since then, slow clinical progression has been observed in both patients.

Severity of disease

Based on the clinical disease severity score, six patients were categorised as having mild disease, two as having moderate disease and five as having severe disease (table 2 and supplementary table E4). Six of the seven patients with either moderate or severe disease were current or former smokers. A correlation was 


\begin{tabular}{|c|c|c|c|c|c|c|c|}
\hline $\begin{array}{l}\text { Patient } \\
\text { ID }\end{array}$ & Nucleotide change & Exon & $\begin{array}{l}\text { Protein } \\
\text { change }\end{array}$ & $\begin{array}{l}\text { Pathogenicity } \\
\text { variant class }\end{array}$ & $\begin{array}{l}\text { Variant } \\
\text { severity }\end{array}$ & $\begin{array}{c}\text { Clinical } \\
\text { disease } \\
\text { severity }^{+}\end{array}$ & Reference \\
\hline \multicolumn{8}{|c|}{ Eight novel allelic variants in SLC34A2 identified in PAM patients } \\
\hline $1 \S$ & $\begin{array}{l}\text { c. } 316 G>A \\
\text { c. } 1238 G>A\end{array}$ & $\begin{array}{c}4 \\
11\end{array}$ & $\begin{array}{l}\text { p.Gly106Arg } \\
\text { p.Trp413Ter }\end{array}$ & I & Moderate & Mild & \\
\hline 2 & c. $560 \mathrm{G}>\mathrm{A}$ & 6 & p.Gly187Glu & III & Moderate & Mild & \\
\hline 3 & c. $646 \mathrm{G}>\mathrm{T}$ & 7 & p.Gly216Ter & II & Severe & Severe & \\
\hline 4 & c. $906 \mathrm{G}>\mathrm{A}$ & 8 & p.Trp302Ter & II & Moderate & Mild & \\
\hline 5 & c. $1136 G>A$ & 10 & p.Cys379Tyr & III & Mild & Mild & \\
\hline 6 & c. $1238 G>A$ & 11 & p.Trp413Ter & II & Moderate & $\begin{array}{c}\text { Not } \\
\text { applicable }\end{array}$ & \\
\hline $7^{f}$ & c.1327delC & 11 & p.Leu443Ter & II & Severe & Severe $\# \#$ & \\
\hline 8 & c.1327delC & 11 & p.Leu443Ter & II & Severe & Severe & \\
\hline 9 & c. $1333+1 G>A$ & $\begin{array}{c}\text { Intron } \\
11\end{array}$ & p.? & II & Severe & Moderate & \\
\hline \multicolumn{8}{|c|}{ Two recurrent allelic variants in SLC34A2 identified in PAM patients } \\
\hline 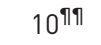 & c. $1390 \mathrm{G}>\mathrm{C}$ & 12 & p.Gly464Arg & । & Moderate & Mild & {$[16]^{++}$} \\
\hline 11 & c. $1390 G>C$ & 12 & p.Gly464Arg & i & Moderate & Mild & {$[16]^{++}$} \\
\hline 12 & c.1402_1404delACC & 12 & p.Thr468del & I & Moderate & Severe & \\
\hline 13 & c.1402_1404delACC & 12 & p.Thr468del & । & Moderate & Severe & [2] \\
\hline 14 & c.1402_1404delACC & 12 & p.Thr468del & I & Moderate & Moderate & [2] \\
\hline
\end{tabular}

PAM: pulmonary alveolar microlithiasis. " : based on the American College of Medical Genetics and Genomics (ACMG) and the Association for Molecular Pathology (AMP) criteria [35] (where I: pathogenic, II: likely pathogenic, III: uncertain significance, IV: likely benign and V: benign); ": predicted effect on $\mathrm{NaPi}-2 \mathrm{~b}$ function based on variant type and localisation within the protein. Variants causing premature truncation or with a presumed crucial localisation within the protein scored one point each. Missense variants and small in-frame deletions scored zero points (where mild: 0 points, moderate: 1 point and severe: 2 points); ${ }^{+}$: disease severity was graded into three groups: mild, moderate and severe, based on a composite measure of the patient's symptoms, lung function, limitation of life due to PAM, signs of advanced disease and disease course (nine parameters in total); $\S_{\text {: }}$ compound heterozygous allele state (all other patients had variants in a homozygous allele state); ${ }^{f}$ : sister of patient $8 ;{ }^{\# \#}$ : deceased; ${ }^{\text {ๆा? }}$ : brother of patient $11{ }^{++}$: IzUMI et al. [16] presented the variant c.1390G $>C$ in heterozygous state together with c.1048+1G>A in a PAM patient (SLC34A2 DNA reference sequence: Ensembl Transcript ID ENST00000382051.7 (GRCh38.p12 assembly)).

found between smoking and disease severity in all patients (Spearman rank correlation coefficient=0.762, $\mathrm{p}=0.003$ ) but this was not statistically significant on excluding the children (Spearman rank correlation coefficient $=0.617, \mathrm{p}=0.057$ ) (see supplementary tables E5 and E6).

\section{Genetic findings}

All 14 patients presented with rare variants in SLC34A2, including eight novel allelic variants (see table 2 and figures 3 and 4). The variants comprised four nonsense, four missense and one splice site variant. In addition, one variant previously described by our group [2], a three nucleotide deletion in exon 12 (c.1402_1404delACC), was found in an unrelated patient. All patients were homozygous for the identified variants, except for patient 1, who was compound heterozygous for a missense variant in exon 4 (c.316G >A) and a nonsense variant in exon 11 (c.1238G $>$ A). The c.1238G $>$ A variant was additionally found in a homozygous state in patient 6 . The mother of patient 2 was found to be a carrier of the same variant as her child and the parents and a sibling of patients 10 and 11 were identified as carriers. None of the carriers had clinical signs or symptoms of PAM.

Eight out of 10 patients were homozygous for an additional variant in SLC34A2 located in exon 13, c.1901A $>$ G, a common variant (rs6448389) with a high but variable allele frequency (81-100\%) in different populations. Another additional variant located in exon 9, c.936T $>$ G, a moderately rare variant (rs112461275) with a reported allele frequency of up to 7\% in some African subpopulations [26], was detected in homozygous form in the French patient originating in Morocco (patient 4). None of these variants were considered as possibly causative for PAM due to their high frequency in the general population.

\section{Classification of the variants}

Variant severity was classified as mild $(n=1)$, moderate $(n=6)$ or severe $(n=3)$, reflecting the predicted effect on $\mathrm{NaPi}-2 \mathrm{~b}$ function based on type and localisation within the protein. Two variants in a compound 


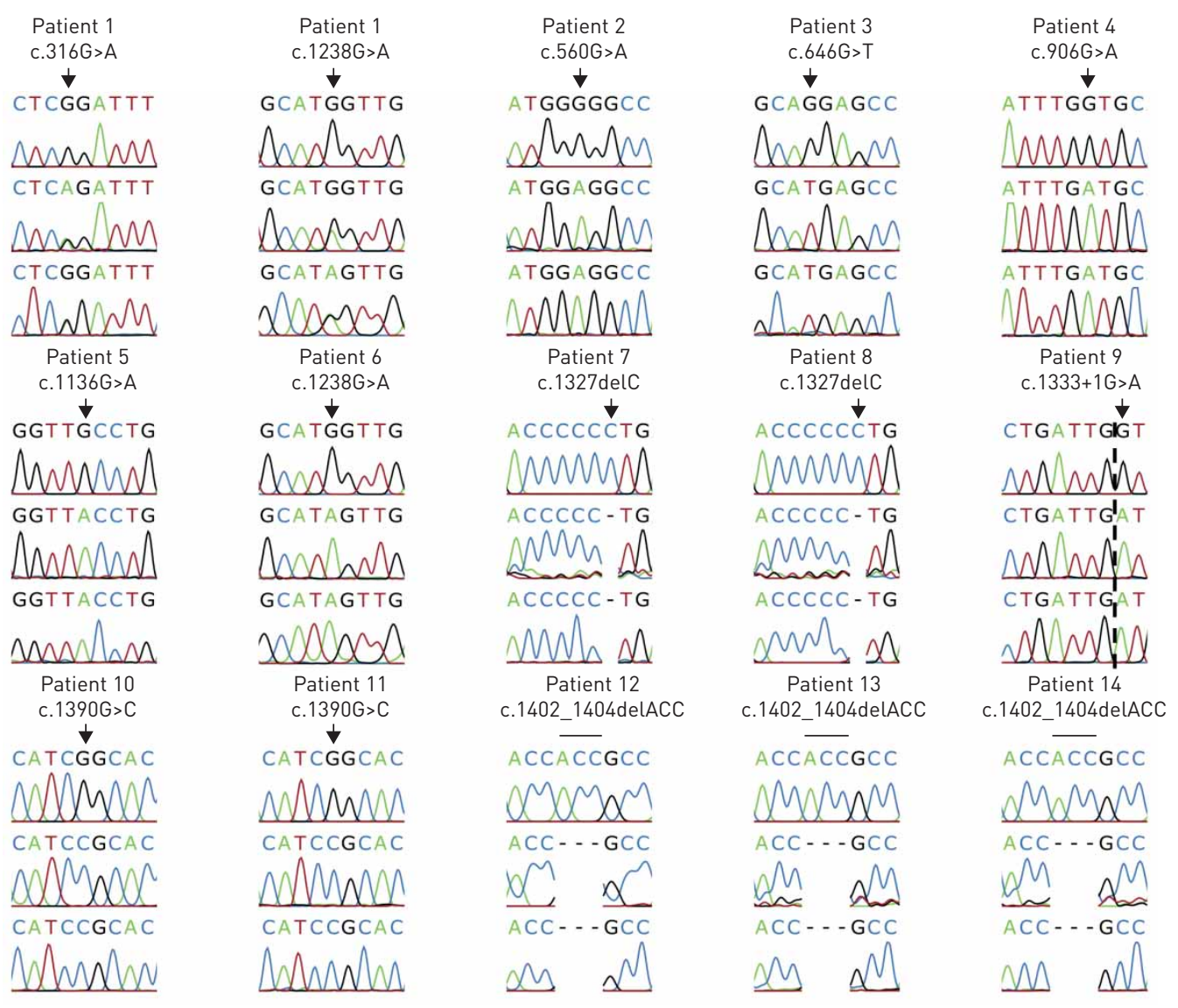

FIGURE 3 DNA sequence analysis of selected regions of SLC34A2 in patients 1-14. Two different allelic variants are found in patient 1 la compound heterozygous state), while all other variants are present in homozygous form. Top row: SLC34A2 DNA reference sequence (Ensembl Transcript ID ENST00000382051.7 (GRCh38.p12 assembly)]. Middle row: forward sequence. Bottom row: reverse sequence. The dotted line represents the border between exon 11 and intron 11. The arrows and bars indicate the locations of the variants.

heterozygous state were given a combined severity score based on the highest predicted value of one of the variants (see table 2 and supplementary table E7). The variants were classified according to the ACMG criteria [35] as Class I (pathogenic $(n=3)$ ), Class II (likely pathogenic $(n=5)$ ) and Class III (uncertain significance $(\mathrm{n}=2))($ table 2$)$.

Four out of 10 of the expected disease-causing allelic variants were absent in the GnomAD, ESP, 1000 Genomes Project and dbSNP databases, and the remaining variants were present in very low frequency and only in the heterozygous state (see supplementary material). In addition, all variants were predicted to be "probably damaging", "deleterious" or "disease causing" by computational prediction tools (see supplementary table E8). Two of the variants (c.1390G>C and c.1402_1404delACC) have previously been described in PAM patients $[2,16]$. In addition, a different nucleotide substitution (c.316G>C) leading to the same amino acid change as in patient 1 (p.Gly106Arg) was previously reported in a Turkish patient [3].

\section{Phenotype and genotype}

Four allelic variants (c.1238G>A, c.1327delC, c.1390G>C and c.1402_1404delACC) were found in more than one patient (patients 1, 6-8 and 10-14). Two different variants (c.1327delC and c.1390G>C) were present in both pairs of siblings (patients 7 and 8 , as well as 10 and 11) and one variant (c.1402_1404delACC) was found in three unrelated patients (patients 12-14). Similar phenotypes were observed in the patients homozygous for the same variants (patients 7 and 8 (c.1327delC), 10 and 11 (c.1390G>C) and 12-14 (c.1402_1404delACC)) (table 2). Both adult sisters (patients 7 and 8) had severe clinical disease. Patient 7 later passed away due to respiratory complications and the other sister was successfully treated with lung transplantation. The young siblings (patients 10 and 11) both had mild clinical disease and were both without any symptoms since the age of four. As most of the patients in the cohort were found with different variants, it was impossible to establish overall specific genotype- 


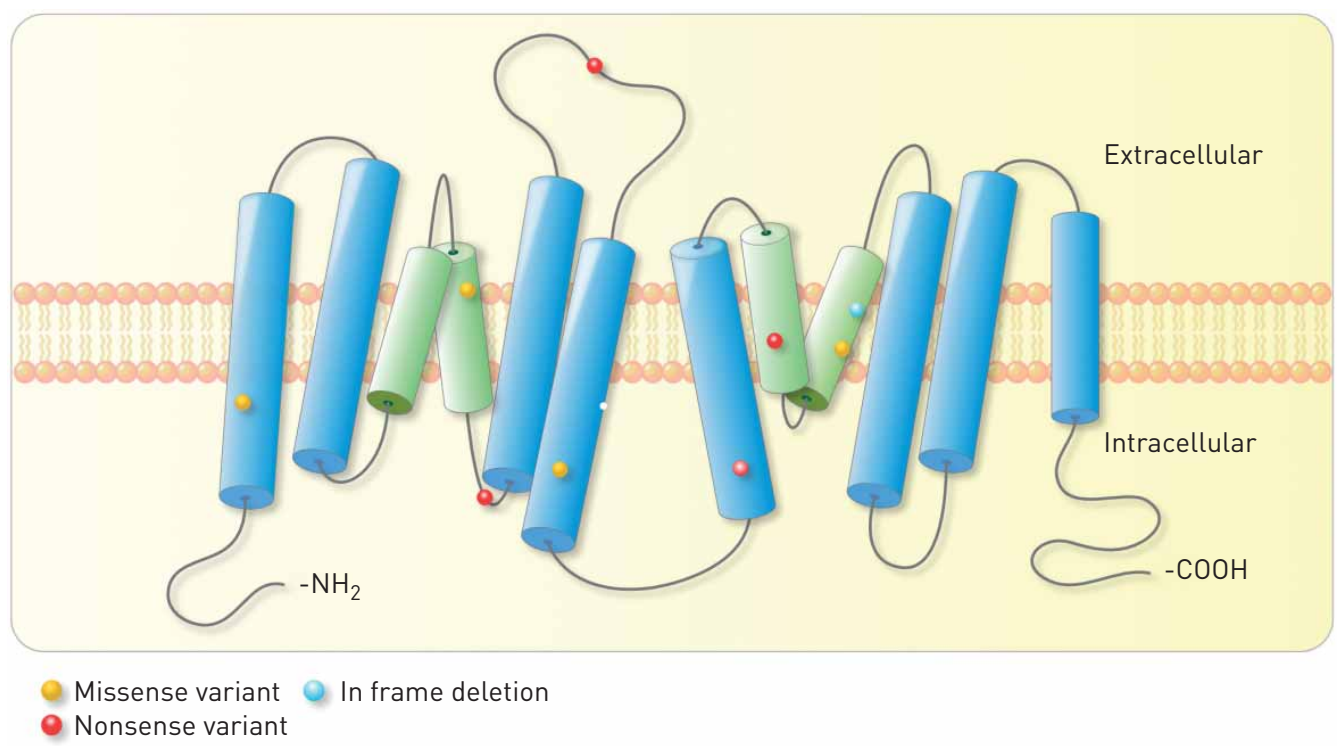

FIGURE 4 Allelic variants in SLC34A2 presented in this report marked on a model of NaPi-2b. All variants, except for the splice site variant (c.1333+1G>A) are shown in the figure as dots. The transmembrane domains (TMDs) 3-4 and 8-9 (green) form the substrate coordination site. Important areas for electrogenicity, regulation and targeting are located in the area between TMDs 4-5, 10-11 and at the C-terminal region [38]. $\mathrm{NaPi}-2 \mathrm{~b}$ belongs to the SLC34 transporter family along with NaPi-2a (SLC34A1) and NaPi-2c (SLC34A3). Significant sequence similarity is present in all SLC34 family members and the eukaryotic NaPi-2 isoforms are predicted to share the same transmembrane topology [39]. The model is made by superimposing human $\mathrm{NaPi}-2 \mathrm{~b}$ on rat NaPi-2a predicted topology and is modified from ForSTER et al. [38] and VIRKKI et al. [40]. The protein sequences used for alignment in Clustal Omega version 1.2.4 [41] were Ensembl Transcript ID ENST00000382051.7 release 92 (Human (GRCh38.p12) assembly) and Ensembl Transcript ID ENSRNOT00000033749.5 (Rat (Rnor_6.0) assembly).

phenotype correlations. However, a correlation was found between disease severity and variant severity (Spearman rank correlation coefficient $=0.629, \mathrm{p}=0.021$ ) (see supplementary table E9).

\section{Discussion}

In this study, we present the genetic analysis of SLC34A2 in 14 PAM patients from six countries. All patients were either homozygous or compound heterozygous for variants in SLC34A2 and eight allelic variants were novel. Both clinical course and age varied greatly among the patients. A correlation was found between disease severity and variant severity based on the variant type and localisation within $\mathrm{NaPi}-2 \mathrm{~b}$. Our results confirm the phenotypic variability of PAM, expand the spectrum of disease-causing variants in SLC34A2 and suggest that disease severity may be associated with the severity of the variants.

PAM is considered to be a monogenic disorder [1]. Genetic variants in SLC34A2 were found in all patients studied. This high detection rate is consistent with the recessive pathogenesis of PAM and it decreases the likeliness of locus heterogeneity. The variants in this report were very likely disease causing since 1) they have not been reported in homozygous form in the general population and all are rare; 2) they were all present in the patients in a homozygous or compound heterozygous state; and 3) they were all predicted to interfere with the structure and/or function of $\mathrm{NaPi}-2 \mathrm{~b}$.

In total, 10 genetic allelic variants were identified in the patients studied. To our knowledge, eight of the alterations identified have not previously been reported and their association with PAM is therefore unknown. Interestingly, four of the novel allelic variants (c.560G>A, c.646G>T, c.1327delC and c.1333 $+1 \mathrm{G}>\mathrm{A}$ ) and two recurrent variants (c.1390G>C [16] and c.1402_1404delACC [2]) are located in functionally critical areas of the protein [38]. The novel splice site variant $(c .1333+1 \mathrm{G}>\mathrm{A})$ is located at the donor splice site immediately after the 3 ' end of exon 11. It might thus lead to a splicing defect that causes either complete intron retention or activation of a cryptic splice site in the intron or in exon 12, leading to a frameshift which results in a premature stop codon.

Three variants in SLC34A2 have previously been functionally investigated in cells $[4,42]$. These studies revealed either a nonfunctional protein (in the case of a 19 amino acid indel and a splice site variant) or signs of reduced phosphate transport function (in the case of a p.Thr192Lys missense variant). Interestingly, the missense variant was located in a presumed functionally critical area of the $\mathrm{NaPi}-2 \mathrm{~b}$ 
protein [38]. One may therefore speculate that location of missense variants in a functionally critical region of the $\mathrm{NaPi}-2 \mathrm{~b}$ protein might, in general, cause changes in structural conformation with possible disruption of its intracellular handling or changes in protein kinetics. This might give rise to proteins which have impaired ability (or are unable) to reach the membrane, thus leading to reduced or removed protein function. However, more functional cell studies are needed to investigate the possible functional impact of different variants.

A recurrent variant in exon 12 (c.1402_1404delACC) seems to be rather frequent in PAM, as it has been identified in three unrelated patients. The variant is located in a repetitive region of four ACCs, which may thus be predisposed to replication errors. Interestingly, the American patient with the variant in question originated from Italy and the variant has previously only been reported in Europeans. The c.1901A>G variant (rs6448389) is common and was therefore expected to be present in most of the patients. More surprising was the detection of the moderately rare variant rs112461275 in patient 4, who originated from Africa. It is noteworthy that this variant is reported with a higher frequency in Africans compared to other populations. However, it is so far unknown whether there exists an association between some specific rare single nucleotide polymorphism (SNP) and the disease itself.

The clinical variability in our patients is in agreement with previous findings [1]. Environmental factors like inflammation and smoking have been suggested to influence both the onset and the course of PAM and thereby partly explain the large clinical variability of the disease $[2,3,19,21]$. In our study, no correlation between smoking and disease severity was found in the adult patients. We acknowledge that this could be due to a relatively small sample size.

Four patients in our cohort presented with calcifications at extrapulmonary sites and two patients (patients 9 and 13) had undergone a valve replacement due to AS. One of these patients (patient 13) was diagnosed with AS, renal stones and gallbladder stones, as well as calcifications in the gastric ventricle consistent with a multisystem disorder. Extrapulmonary calcifications in PAM might be caused by dysfunctional $\mathrm{NaPi}-2 \mathrm{~b}$ in the tissues affected $[1,2,37]$.

One patient was treated with lung transplantation, which is the only known effective treatment in PAM as systemic corticosteroids and therapeutic bronchoalveolar lavage (BAL) with saline are considered to be noneffective. A possible beneficial effect of bisphosphonates and sodium thiosulfate (STS) remains to be explored [1,36]. Since variable degrees of fibrosis and inflammation of the pulmonary interstitium are accompanying features of calcification in advanced disease [43], treatment with antifibrotic agents could possibly be of relevance in some cases. To the best of our knowledge, antifibrotic treatment has so far not been reported in PAM.

A patient in our cohort was misclassified with sarcoidosis prior to the diagnosis of PAM; however, while it is uncommon, multiple micronodular calcifications can be seen in sarcoidosis [44]. Pulmonary calcification and ossification are associated with a variety of pulmonary and systemic diseases, such as metastatic calcification due to chronic renal failure, dystrophic calcification due to granulomatous disorders, DNA viruses, parasitic infections, pulmonary amyloidosis and various forms of pulmonary ossification (e.g. idiopathic dendriform diffuse pulmonary ossification) [44, 45]. Different imaging techniques are used to recognise specific patterns and if it is not conclusive, a biopsy is needed for the diagnosis. The histopathology of PAM is characteristic, in which concentric laminated calcium phosphate concretions are present inside the alveoli, whereas calcifications are found in the interstitial or vascular compartments in metastatic and dystrophic calcifications [44]. If histological investigation is not clear, the diagnosis of PAM may be confirmed by genetic evaluation of the SLC34A2 gene.

No genotype-phenotype correlation has been described in patients with PAM [1]. However, only a few variants in a relatively small number of patients have so far been reported and the clinical data on patients is based on case reports with variable levels of detail. In our report, one of the variants was found in three apparently unrelated patients and all of them presented with moderate to severe disease. However, two of these patients are now in their sixties and the clinical severity might just reflect the slowly progressive nature of the disease (i.e. a survivor effect). On the other hand, the third patient was only an adolescent when significant symptoms appeared. Given most of the variants in this report were detected only once, no overall specific genotype-phenotype correlations were established. However, an interesting correlation between disease severity and variant severity was found.

We categorised the severity of disease based on a composite measure of different clinical parameters; however, we acknowledge this categorisation is subject to bias and standardised methods and validation are still lacking. Furthermore, we acknowledge the possibility of confounding due to age difference and variation in smoking status amongst the patients. The functional effects of variants in SLC34A2 are not well investigated so far, and the classification and evaluation of different genotypes in this report are 
therefore determined merely on a theoretical basis. Therefore, the findings in this study need to be confirmed and further addressed in larger patient series and functional studies. The main strength of this report is the presentation of both clinical and genetic data from a relatively large cohort of PAM patients from several countries. Furthermore, all genetic analyses were carried out in the same laboratory.

In conclusion, we detected eight novel allelic variants in SLC34A2 in 14 PAM patients and variants were found in all patients studied. Our findings emphasise a significant role of SLC34A2 in PAM and, in addition, expand the variant spectrum of SLC34A2 in PAM patients. Data from this report suggest that an association may exist between severity of the phenotype and the variants. Functional experiments with variants in SLC34A2 identified in the patients, combined with clinical studies, are warranted to understand the pathophysiology of PAM and to elucidate possible genotype-phenotype correlations. Our hope is that, in the future, improved knowledge may lead to specific pharmacological and nonpharmacological treatment strategies.

Acknowledgements: The authors wish to thank Anne Hedemand and Tanja Stenshøj Østergaard (Dept of Biomedicine, Aarhus University, Aarhus, Denmark) for excellent technical support and to the patients who contributed to the research.

Conflict of interest: Å.L.M. Jönsson has nothing to disclose. E. Bendstrup has nothing to disclose. S. Mogensen has nothing to disclose. E.J. Kopras reports grants from the NHLBI (R01HL127455 and U54HL127672) during the conduct of the study. F.X. McCormack reports grants from the NIH during the conduct of the study. I. Campo has nothing to disclose. F. Mariani has nothing to disclose. A. Escribano-Montaner has nothing to disclose. A.M. Holm has nothing to disclose. M.M. Martinez-Colls has nothing to disclose. G. Pintos-Morell reports personal fees for lectures from Shire, honoraria for consultancy from Sanofi-Genzyme and Alexion, and personal fees for travel and consultancy from Kyowa-Kirin, outside the submitted work. C. Taillé has been an investigator in trials and received personal fees for consultancy and advisory board work from AstraZeneca, GlaxoSmithKline, Novartis, Sanofi and Roche, received personal fees for consultancy and advisory board work from Teva and Genzyme, and has been an investigator in trials from Boehringer Ingelheim, outside the submitted work. B. Crestani reports personal fees for lectures and nonfinancial support for meeting attendance from AstraZeneca, grants, personal fees for lectures and nonfinancial support for meeting attendance from Boehringer Ingelheim and Roche, personal fees for lectures and consultancy, as well as nonfinancial support for meeting attendance from Sanofi, personal fees for advisory board work from Genzyme and grants from MedImmune, outside the submitted work. O. Hilberg has nothing to disclose. J. Hvarregaard Christensen has nothing to disclose. U. Simonsen has nothing to disclose.

Support statement: This work was supported by unrestricted grants from the Graduate School of Health Aarhus University, MEMBRANES Aarhus University, Central Denmark Region, Fonden til Lægevidenskabens Fremme A.P. Møller og Hustru Chastine Mc-Kinney Møllers Fond til almene Formaal, the Novo Nordisk Foundation (grant number NNF60C0023284), and the LAM Foundation. Funding information for this article has been deposited with the Crossref Funder Registry.

\section{References}

1 Castellana G, Castellana G, Gentile M, et al. Pulmonary alveolar microlithiasis: review of the 1022 cases reported worldwide. Eur Respir Rev 2015; 24: 607-620.

2 Jönsson ÅL, Simonsen U, Hilberg O, et al. Pulmonary alveolar microlithiasis: two case reports and review of the literature. Eur Respir Rev 2012; 21: 249-256.

3 Corut A, Senyigit A, Ugur SA, et al. Mutations in SLC34A2 cause pulmonary alveolar microlithiasis and are possibly associated with testicular microlithiasis. Am J Hum Genet 2006; 79: 650-656.

4 Huqun, Izumi S, Miyazawa $\mathrm{H}$, et al. Mutations in the SLC34A2 gene are associated with pulmonary alveolar microlithiasis. Am J Respir Crit Care Med 2007; 175: 263-268.

5 SLC34A2 solute carrier family 34 member 2 [homo sapiens (human)] (Gene ID: 10568). National Center for Biotechnology Information, U.S. National Library of Medicine, Bethesda, MD, USA. www.ncbi.nlm.nih.gov/gene/? term=10568 Date last accessed: March 29, 2019. Date last updated: December 10, 2019.

6 The UniProt C. UniProt: the universal protein knowledgebase. Nucleic Acids Res 2017; 45: D158-D169.

$7 \quad$ Zerbino DR, Achuthan P, Akanni W, et al. Ensembl 2018. Nucleic Acids Res 2018; 46: D754-D761.

8 Traebert M, Hattenhauer O, Murer H, et al. Expression of type II Na-P(i) cotransporter in alveolar type II cells. Am J Physiol 1999; 277: L868-L873.

9 Poelma DL, Ju MR, Bakker SC, et al. A common pathway for the uptake of surfactant lipids by alveolar cells. Am J Respir Cell Mol Biol 2004; 30: 751-758.

10 Saito A, Nikolaidis NM, Amlal H, et al. Modeling pulmonary alveolar microlithiasis by epithelial deletion of the $\mathrm{Npt} 2 \mathrm{~b}$ sodium phosphate cotransporter reveals putative biomarkers and strategies for treatment. Sci Transl Med 2015; 7: 313ra181.

11 Vismara MF, Colao E, Fabiani F, et al. The sodium-phosphate co-transporter SLC34A2, and pulmonary alveolar microlithiasis: presentation of an inbred family and a novel truncating mutation in exon 3. Respir Med Case Rep 2015; 16: 77-80.

12 Dandan S, Yuqin C, Wei L, et al. Novel deletion of SLC34A2 in Chinese patients of PAM shares mutation hot spot with fusion gene SLC34A2-ROS1 in lung cancer. J Genet 2018; 97: 939-944.

13 Gaber K, Najem S, Bader O, et al. New mutation of SCL34A2 gene in a family with pulmonary alveolar microlithiasis in Libya. Chest 2012; 142: 4 Suppl., 439A.

14 Alastal A, Yamin H. Pulmonary alveolar microlithiasis caused by two homozygous mutations. Am J Respir Crit Care Med 2017; 195: A3438. 
15 Ellison J. Novel human pathological mutations. Gene symbol: SLC34A2. Disease: pulmonary alveolar microlithiasis. Hum Genet 2009; 125: 333.

16 Izumi H, Kurai J, Kodani M, et al. A novel SLC34A2 mutation in a patient with pulmonary alveolar microlithiasis. Hum Genome Var 2017; 4: 16047.

17 Stokman L, Nossent EJ, Grunberg K, et al. A case of pulmonary alveolar microlithiasis associated with a homozygous $195 \mathrm{~kb}$ deletion encompassing the entire SLC34A2 gene. Clin Case Rep 2016; 4: 412-415.

18 Ishihara Y, Hagiwara K, Zen K, et al. A case of pulmonary alveolar microlithiasis with an intragenetic deletion in SLC34A2 detected by a genome-wide SNP study. Thorax 2009; 64: 365-367.

19 Wang $\mathrm{H}$, Yin $\mathrm{X}, \mathrm{Wu} \mathrm{D}$, et al. SLC34A2 gene compound heterozygous mutation identification in a patient with pulmonary alveolar microlithiasis and computational 3D protein structure prediction. Meta Gene 2014; 2: 557-564.

20 Yin X, Wang H, Wu D, et al. SLC34A2 gene mutation of pulmonary alveolar microlithiasis: report of four cases and review of literatures. Respir Med 2013; 107: 217-222.

21 Proesmans M, Boon M, Verbeken E, et al. Pulmonary alveolar microlithiasis: a case report and review of the literature. Eur J Pediatr 2012; 171: 1069-1072.

22 Ma T, Ren J, Yin J, et al. A pedigree with pulmonary alveolar microlithiasis: a clinical case report and literature review. Cell Biochem Biophys 2014; 70: 565-572.

23 Simon CT, Lewis TC, Neemuchwala F, et al. Pulmonary alveolar microlithiasis: a case report with a novel mutation in the SLC34A2 gene and review of the literature. Hum Path: Case Rep 2018; 13: 33-35.

24 Lek M, Karczewski KJ, Minikel EV, et al. Analysis of protein-coding genetic variation in 60,706 humans. Nature 2016; 536: 285-291.

25 Exome Variant Server, NHLBI GO Exome Sequencing Project (ESP), Seattle, WA, USA. http://evs.gs.washington. edu/EVS/ Date last accessed: March 2019.

261000 Genomes Project Consortium, Auton A, Brooks LD, et al. A global reference for human genetic variation. Nature 2015; 526: 68-74.

27 Sherry ST, Ward MH, Kholodov M, et al. dbSNP: the NCBI database of genetic variation. Nucleic Acids Res 2001; 29: $308-311$.

28 National Center for Biotechnology Information, U.S. National Library of Medicine, Bethesda, MD, USA. www. ncbi.nlm.nih.gov Date last accessed: March 29, 2019.

29 Stenson PD, Mort M, Ball EV, et al. The Human Gene Mutation Database: towards a comprehensive repository of inherited mutation data for medical research, genetic diagnosis and next-generation sequencing studies. Hum Genet 2017; 136: 665-677.

30 Schwarz JM, Cooper DN, Schuelke M, et al. MutationTaster2: mutation prediction for the deep-sequencing age. Nat Methods 2014; 11: 361-362.

31 Mi H, Huang X, Muruganujan A, et al. PANTHER version 11: expanded annotation data from Gene Ontology and Reactome pathways, and data analysis tool enhancements. Nucleic Acids Res 2017; 45: D183-D189.

32 Adzhubei IA, Schmidt S, Peshkin L, et al. A method and server for predicting damaging missense mutations. Nat Methods 2010; 7: 248-249.

33 Choi Y, Chan AP. PROVEAN web server: a tool to predict the functional effect of amino acid substitutions and indels. Bioinformatics 2015; 31: 2745-2747.

34 Desmet FO, Hamroun D, Lalande M, et al. Human Splicing Finder: an online bioinformatics tool to predict splicing signals. Nucleic Acids Res 2009; 37: e67.

35 Richards S, Aziz N, Bale S, et al. Standards and guidelines for the interpretation of sequence variants: a joint consensus recommendation of the American College of Medical Genetics and Genomics and the Association for Molecular Pathology. Genet Med 2015; 17: 405-424.

36 Taille C, Debray MP, Danel C, et al. Calcium-solubilizing sodium thiosulfate failed to improve pulmonary alveolar microlithiasis: evaluation of calcium content with CT scan. Respir Med Res 2019; 75: 10-12.

37 Jönsson ÅL, Hilberg O, Bendstrup EM, et al. SLC34A2 gene mutation may explain comorbidity of pulmonary alveolar microlithiasis and aortic valve sclerosis. Am J Respir Crit Care Med 2012; 185: 464.

38 Forster IC, Hernando N, Biber J, et al. Phosphate transporters of the SLC20 and SLC34 families. Mol Aspects Med 2013; 34: 386-395

39 Forster IC, Hernando N, Biber J, et al. Phosphate transport kinetics and structure-function relationships of SLC34 and SLC20 proteins. Curr Top Membr 2012; 70: 313-356.

40 Virkki LV, Biber J, Murer H, et al. Phosphate transporters: a tale of two solute carrier families. Am J Physiol Renal Physiol 2007; 293: F643-F654.

41 Sievers F, Wilm A, Dineen D, et al. Fast, scalable generation of high-quality protein multiple sequence alignments using Clustal Omega. Mol Syst Biol 2011; 7: 539.

$42 \mathrm{Ma}$ T, Qu D, Yan B, et al. Effect of SLC34A2 gene mutation on extracellular phosphorus transport in PAM alveolar epithelial cells. Exp Ther Med 2018; 15: 310-314.

43 Prakash UB, Barham SS, Rosenow EC, 3rd, et al. Pulmonary alveolar microlithiasis. A review including ultrastructural and pulmonary function studies. Mayo Clin Proc 1983; 58: 290-300.

44 Chan ED, Morales DV, Welsh CH, et al. Calcium deposition with or without bone formation in the lung. Am Respir Crit Care Med 2002; 165: 1654-1669.

45 Slabbynck H, de Beukelaar T, De Surgeloose D, et al. Predominant dendriform pulmonary ossification in a usual interstitial pneumonia-like distribution: to be distinguished from idiopathic pulmonary fibrosis. Sarcoidosis Vasc Dif Lung Dis 2017; 34: 251-256. 